(C) 2021, The Authors. Published by Elsevier Inc. and Fass Inc. on behalf of the American Dairy Science Association ${ }^{\circledR}$. This is an open access article under the CC BY-NC-ND license (http://creativecommons.org/licenses/by-nc-nd/4.0/).

\title{
Short communication: Effects of different blood buffers administered in electrolyte solution to grain-fed veal calves experiencing diarrhea
}

\author{
D. R. Wood, ${ }^{1 *}$ 다. R. M. Blome, ${ }^{1}$ L. C. Ribeiro, ${ }^{1}$ A. J. Keunen, ${ }^{2}$ B. W. Keunen, ${ }^{2}$ G. W. Smith, ${ }^{3}{ }^{\circ}$ \\ and D. L. Renaud ${ }^{4}$ (1) \\ ${ }^{1}$ Animix, Juneau, WI 53039 \\ ${ }^{2}$ Mapleview Agri, Palmerston, Ontario NOG 2PO, Canada \\ ${ }^{3}$ Department of Population, Health, and Pathology, College of Veterinary Medicine, North Carolina State University, Raleigh 27607 \\ ${ }^{4}$ Department of Population Medicine, University of Guelph, Guelph, Ontario N1S 2W1, Canada
}

\section{ABSTRACT}

Calf diarrhea can commonly lead to dehydration and metabolic acidosis due to the loss of fluid and electrolytes. The objective of this randomized clinical trial was to examine differences between treating male dairy calves experiencing diarrhea with either a basic bicarbonate electrolyte powder (BBP) composed of sodium bicarbonate $(50.7 \mathrm{mmol} / \mathrm{L})$; a mixed buffer powder (MBP) including sodium bicarbonate (33.8 $\mathrm{mmol} / \mathrm{L})$, sodium citrate $(8.4 \mathrm{mmol} / \mathrm{L})$, sodium acetate $(6.3 \mathrm{mmol} / \mathrm{L})$, and potassium citrate $(1.9 \mathrm{mmol} / \mathrm{L})$; or a liquid electrolyte (HAL) composed of sodium acetate $(50.1 \mathrm{mmol} / \mathrm{L})$. All 3 electrolyte solutions were standardized to provide $50 \mathrm{mmol} / \mathrm{L}$ blood buffers and a similarly strong ion difference $(74.4,74.9$, and 82.6 $\mathrm{mEq} / \mathrm{L}$ for $\mathrm{BBP}, \mathrm{MBP}$, and HAL, respectively). Holstein male calves $(\mathrm{n}=80)$ were sourced from auction barns or local farms and delivered in 1 batch to the research facility. Calves were housed in individual pens and fed a $24 \%$ crude protein and $17 \%$ fat calf milk replacer (CMR) twice daily. Starter grain and water were offered ad libitum. Calves were randomly enrolled in 1 of the 3 treatments when experiencing either 2 consecutive days of a fecal score of 2 (runny, spreads easily) or $1 \mathrm{~d}$ with a fecal score of 3 (liquid devoid of solid material). Calves were blocked by the different enrollment criteria. The respective electrolyte solution was administered via esophageal tube $1 \mathrm{~h}$ after feeding CMR until the fecal score returned to 0 (normal consistency) or 1 (semiformed or pasty). Blood gas measurements were taken at 1,8 , and $24 \mathrm{~h}$ post the initial electrolyte feeding, and weight was measured at 1, 2, 7, 14, and $28 \mathrm{~d}$ postenrollment. Mixed repeated measure linear regression models were built to assess the effect

Received March 13, 2020.

Accepted August 24, 2020.

*Corresponding author: biowood10@gmail.com that the electrolyte solutions had on the blood gas measurements and body weight. A total of 45 calves were enrolled in the trial with 14,16 , and 15 calves randomly assigned to the MBP, HAL, and BBP groups, respectively. As compared with $\mathrm{BBP}, \mathrm{MBP}$ increased blood $\mathrm{CO}_{2}$ at 8 and $24 \mathrm{~h}$, increased bicarbonate at 24 $\mathrm{h}$, increased base excess at 8 and $24 \mathrm{~h}$, and increased anion gap at $24 \mathrm{~h}$. Calves in the BBP and HAL groups noted more severe eye recession when compared with the MBP group. Average daily gain did not differ between treatments at any time point. Although a severe dehydration challenge was not present, which should be considered a limitation of the study, MBP improved the acid-base status of calves compared with BBP, whereas HAL performed similarly to MBP.

Key words: diarrhea, electrolytes, blood buffers, calf

\section{Short Communication}

Neonatal diarrhea is a common disease in young calves. Early and aggressive oral electrolyte therapy on the onset of diarrhea in calves is critical in replenishing fluids and correcting metabolic acidosis (Smith and Berchtold, 2014). Traditionally, oral electrolyte solutions (OES) have contained sodium bicarbonate as an alkalinizing agent to correct metabolic acidosis because it has been shown to be very effective (Naylor et al., 1990). However, replacing sodium bicarbonate in an OES may have some appeal as several disadvantages have been identified when using high concentrations of bicarbonate in electrolyte products. High concentrations of bicarbonate alkalinize the abomasum, which could lead to a higher number of coliform bacteria that reach the small intestine, thus increasing the severity, duration, and mortality risk associated with diarrhea in calves (Smith et al., 2012). There is also concern that bicarbonate may interfere with milk clotting in the abomasum as previous studies identified that OES with a bicarbonate concentration $>40 \mathrm{mEq} / \mathrm{L}$ has negative effects on milk clotting (Miyazaki et al., 2019). 
Newer OES formulations contain acetate, propionate, and citrate, which have been shown to have similar alkalinizing effects to bicarbonate (Doré et al., 2019). They are considered bicarbonate precursors because they produce water and bicarbonate ions when metabolized. This metabolic process appears to still function efficiently in calves with severe diarrhea (Bachmann et al., 2009). In addition, metabolizable bases such as acetate, citrate, and propionate do not increase abomasal $\mathrm{pH}$ or interfere with milk digestion (Constable et al., 2009), mitigating some of the negative effects of using bicarbonate. The use of acetate and propionate can also have an additional benefit where they act to facilitate sodium and water absorption in the intestine and colon, which does not occur with bicarbonate (Smith, 2009). Thus, OES with acetate or propionate or a mixture of buffers are likely preferable to those that use only high concentrations of bicarbonate. However, no comparisons have been made between multibuffer OES and traditional basic OES formulated with relatively inexpensive sodium bicarbonate as the sole blood buffer.

The primary objectives of this study were to test the effects of different electrolyte solutions on hydration status and blood gas measurements in male dairy calves experiencing diarrhea. Evaluation of the effect of electrolyte solutions on weight gain and days to resolution of an abnormal fecal consistency score were secondary objectives. Our hypothesis was that multisource buffers would lead to improved hydration status, weight gain, and blood gas measurements including blood $\mathrm{pH}$ when compared with a single-source buffer composed of only sodium bicarbonate. However, we anticipated that the resolution of an abnormal fecal consistency score would not be different by treatment groups.

The randomized clinical trial was conducted in fall of 2019 at Mapleview Agri Ltd., a grain-fed veal facility used for calf research located in southwestern Ontario, Canada. Calves were sourced from local dairy farms or auction facilities in Ontario. Calves arrived at the facility in a single batch of 80 male Holstein calves.

Upon arrival to the facility, whole blood was collected, and serum was separated and placed on the measuring surface of the digital refractometer (MISCO PA202, MISCO, Solon, OH) to estimate serum total protein (STP). A STP of $<5.1 \mathrm{~g} / \mathrm{dL}$ was used as the threshold for determination of failure of passive transfer of immunity (FPT; Renaud et al., 2018). Calves were individually housed in $1-\mathrm{m}^{2}$ individual stalls for the milk feeding period. Calves were bucket-fed milk replacer (24\% all-milk protein partially from skim-milk, and $17 \%$ fat with no feed additives) at a daily feeding rate of $4 \mathrm{~L}$, increasing to $7 \mathrm{~L}$ at wk 4 (split evenly between a.m. and p.m.), and then gradually decreasing to complete wean at wk 8. Calves had ad libitum access to water and grain.

Calves were enrolled only in the first 3 wk following arrival to the facility. Enrollment was based on fecal score, with each calf being scored once daily by 1 of 2 trained personnel. Calves were enrolled in the study if identified with 2 consecutive days with a fecal score of 2 (runny, spreads easily) or a single day with a fecal score of 3 (liquid, devoid of solid material; McGuirk, 2008; Medrano-Galarza et al. 2018). A different randomization sheet was used for calves that were enrolled with 2 consecutive days of a fecal score of 2 and those enrolled with a fecal score of 3 . Calves on either randomization sheet were randomized in blocks of 6 according to the RAND command in Microsoft Excel (Microsoft Corp., Redmond, WA). When enrolled, calves were randomly assigned to receive 1 of 3 electrolyte solutions administered in $1.9 \mathrm{~L}$ of warm water, either (1) a basic bicarbonate powder (BBP) composed of $50.7 \mathrm{mmol} / \mathrm{L}$ sodium bicarbonate administered as a 75-g dose, (2) mixed buffers powder (MBP) composed of $33.8 \mathrm{mmol} / \mathrm{L}$ sodium bicarbonate, $8.4 \mathrm{mmol} / \mathrm{L}$ sodium citrate, $6.3 \mathrm{mmol} / \mathrm{L}$ sodium acetate, and $1.9 \mathrm{mmol} / \mathrm{L}$ potassium citrate and administered as a 113-g dose, or (3) high acetate liquid (HAL) composed of $50.1 \mathrm{mmol} / \mathrm{L}$ sodium acetate administered as a $150-\mathrm{mL}$ dose. A detailed composition of the experimental electrolyte products is available in Table 1. Calves were fed the electrolyte solution twice daily via an esophageal tube feeder $1 \mathrm{~h}$ following milk feeding until resolution of abnormal fecal score (fecal score of 0 or 1 ).

Following enrollment, calves were fecal-scored daily for at least 14 consecutive days. Calves were weighed using a digital scale at enrollment and 1, 2, 7, 14, and $28 \mathrm{~d}$ following enrollment. Blood was collected from the jugular vein with a 20-gauge 1-inch needle into a lithium heparin vacuum tube (BD Vacutainer, Becton, Dickson and Company, Franklin Lakes, NJ). The Vacutainer tube has been show in previous studies to have a minimal effect on $\mathrm{pH}$, bicarbonate, and total $\mathrm{CO}_{2}$ when compared to syringe collected samples, however, vacutainer tube collected samples will have a slight decrease in partial pressure of $\mathrm{CO}_{2}$ concentrations (Noël et al., 2010). Immediately following collection, the samples were tested using an Abaxis Vetscan i-STAT 1 Handheld Analyzer (Abaxis, Union City, CA) to measure $\mathrm{Na}$, hemoglobin, $\mathrm{K}$, partial pressure of $\mathrm{CO}_{2}, \mathrm{pH}$, total $\mathrm{CO}_{2}$, hematocrit, $\mathrm{HCO}_{3}$, base excess, BUN, anion gap, glucose, and $\mathrm{Cl}$. Blood was taken at enrollment and 1, 8 , and $24 \mathrm{~h}$ following enrollment. Clinical metrics associated with dehydration were scored using subjective dehydration, attitude, eyeball recession, and skin tent measures (Table 2; Smith, 2009) as ordinal outcomes at the time points when blood was collected, as well 
Table 1. Properties of the electrolyte solutions

\begin{tabular}{lccc}
\hline Nutrient & Basic bicarbonate powder & Mixed buffer powder & High acetate liquid \\
\hline Sodium $(\mathrm{mmol} / \mathrm{L})$ & 119.8 & 143.1 & 130.4 \\
Potassium (mmol/L) & 29.1 & 29.1 & 28.7 \\
Chloride (mmol/L) & 97.3 & 97.3 & 64.9 \\
Dextrose (mmol/L) & 105.8 & 101.5 & 61.6 \\
Glycine (mmol/L) & 7.57 & 45.4 & 45.4 \\
Na bicarbonate (mmol/L) & 50.7 & 33.8 & 0 \\
Na citrate (mmol/L) & 0 & 8.4 & 50.1 \\
Na acetate (mmol/L) & 0 & 6.3 & 0 \\
K citrate (mmol/L) & 0 & 1.9 & 50.1 \\
Total buffers (mmol/L) & 50.7 & 50.4 & 82.6 \\
Strong ion difference (mEq) & 74.4 & 11.9 & 150 \\
Dosage per 1.9 L (g) & 75.5 & 535 & 523 \\
Osmolarity ${ }^{1}(\mathrm{mmol} / \mathrm{L})$ & 371 & & \\
${ }^{1}$ Advanced Instruments (Norwood, MA) osmometer, model no. 3320, using freezing point depression method \\
technology.
\end{tabular}

as 48 and $72 \mathrm{~h}$ following enrollment. Antimicrobial treatment and supportive therapy (nonsteroidal antiinflammatory medications and intravenous fluids) were recorded for each calf.
Those that were responsible for calf enrollment, outcome assessment, and statistical analysis were blinded to the treatment groups; however, research personnel responsible for administration of the treatments were

Table 2. Subjective clinical dehydration indicators measured at enrollment and 1, 8, 24, 48, and $72 \mathrm{~h}$ following enrollment by treatment group measured on 45 male Holstein dairy calves reported as the number of calves in the respective group

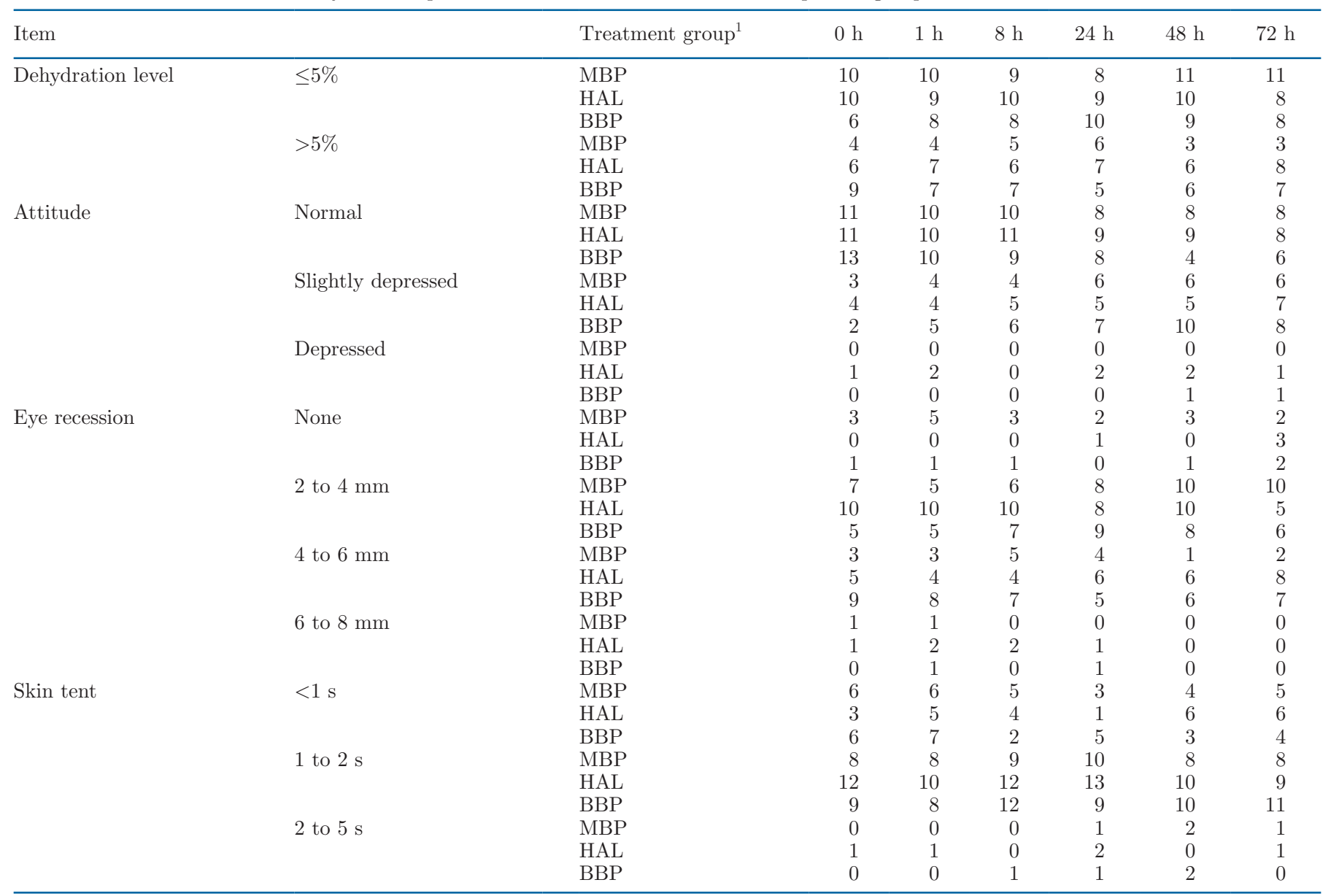

${ }^{1} \mathrm{MBP}=$ mixed buffer powder; HAL $=$ high acetate liquid; $\mathrm{BBP}=$ basic bicarbonate powder. 
not blinded. A formal sample size calculation was not completed, and the sample size was based on the resources that were available.

All statistical analyses were conducted in Stata 14 (StataCorp, College Station, TX). Descriptive statistics were generated on all explanatory variables in the data set with a one-way ANOVA with a Tukey posthoc correction used to compare groups with respect to continuous variables measured at arrival and ADG in the different periods, whereas a $\chi^{2}$ test was used to evaluate differences between categorical variables.

To investigate time to resolution of an abnormal fecal score, a Cox proportional hazards model was used. Resolution was defined as having $1 \mathrm{~d}$ with a fecal score $<2$. Mixed repeated measure linear regression models were built to assess the effect that the electrolyte solutions had on the blood gas measurements and body BW. For the subjective clinical measurements scored, mixed repeated measures ordered logistic regression analysis was conducted. In each of the repeated measures models, the source of the calves, concentration of STP, treatment group, time, and an interaction term of time and treatment group were tested in univariable analysis, whereas for the Cox proportional hazards model, source of the calves, the concentration of STP, and BW at enrollment were offered. Univariable regression models were constructed to screen for variables that were unconditionally associated with the outcome using a liberal $\alpha$-value of 0.2 (Dohoo et al., 2010). Risk factors that had univariate associations $(P<0.2)$ were subsequently offered to a multivariable model through a manual backward stepwise process. Model fit and the presence of outliers was evaluated for each individual model.

A total of 45 calves were enrolled in the trial with 14, 16 , and 15 calves randomly assigned to the MBP, HAL, and BBP groups, respectively. The mean weight of the calves when enrolled was $49.5 \pm 4.0 \mathrm{~kg}$ (mean $\pm \mathrm{SD}$ ) and was not different between the groups $(P=0.96)$. The average STP concentration upon arrival at the research barn was $5.82 \mathrm{~g} / \mathrm{dL}(\mathrm{SD}=0.71)$ with $13.3 \%$ of calves having FPT. Serum total protein concentrations were not different between the groups $(P=0.88)$; however, the BBP group (33.3\%) had a higher level of FPT compared with MBP $(7.1 \%)$ and HAL $(0 \% ; P$ $=0.02)$. No differences were found between treatment groups with respect to source $(P=0.83)$. Thirty-three of the calves were enrolled with 2 consecutive days with a fecal score of 2 , whereas 12 calves were enrolled with $1 \mathrm{~d}$ of a fecal score of 3 . The number of days in the research barn prior to enrollment varied from 3 to 12 with a mean of $7.4 \mathrm{~d}$. The enrollment criteria were not different between the groups $(P=0.98)$. Additionally, no differences between the groups at enrollment were found with respect to the level of dehydration or the measured blood measurements.

Time for diarrhea to return to a fecal score of $<2$ after enrollment had a mean of $3.9 \pm 2.8 \mathrm{~d}, 3.0 \pm 1.7$ $\mathrm{d}$, and $4.7 \pm 3.1 \mathrm{~d}$ in the MBP, HAL, and BBP groups, respectively. No differences between the treatment groups with respect to diarrhea resolution were found. Fecal samples were randomly collected from 4 calves within each group, and Cryptosporidium parvum, rotavirus $\mathrm{A}$, and coronavirus were found in $75 \%, 67 \%$, and $50 \%$ of samples, respectively. No differences between the groups were found with respect to antimicrobial treatment for diarrhea $(P=0.85)$ or respiratory disease $(P=0.59)$. Incidence of milk-replacer refusals occurred in 1,0 , and 4 calves, totaling $1.04,0$, and $1.69 \mathrm{~kg}$ for $\mathrm{MBP}, \mathrm{HAL}$, and $\mathrm{BBP}$, respectively. With respect to BW, the HAL group had a greater BW compared with MBP on d 2 after enrollment $(P=0.04)$; however, no other significant differences were found at any other time points.

Table 3 describes the blood gas measurements by different time points with most of the measurements remaining within their respective reference range. For each of the blood gas measurements, a mixed repeated measures linear regression model was constructed, and solely the treatment groups, time, and their interaction term remained significant. The concentrations of sodium in MBP were higher than the BBP group at $1 \mathrm{~h}$ $(P=0.04), 8 \mathrm{~h}(P<0.001)$, and $24 \mathrm{~h}(P<0.001)$ after enrollment. With respect to potassium, the concentrations were lower at $1 \mathrm{~h}$ after enrollment in the MBP group when compared with the HAL group $(P<0.001)$. Chloride was lower in the HAL group than in the MBP group throughout the experimental period (coefficient $=-2.53 \mathrm{mmol} / \mathrm{L} ; P=0.03 ; 95 \% \mathrm{CI}=-4.81$ to -0.26$)$. Total $\mathrm{CO}_{2}$ was lower in $\mathrm{BBP}$ at $8 \mathrm{~h}(P=0.03)$ and 24 $\mathrm{h}(P=0.01)$ after enrollment compared with MBP. The HAL group had higher concentrations of BUN compared with MBP at $24 \mathrm{~h}$ after enrollment $(P=0.005)$. At 1 $\mathrm{h}$ following enrollment, HAL had a lower $\mathrm{pCO}_{2}$ than MBP $(P=0.01)$, whereas at $8 \mathrm{~h}$ following enrollment, the $\mathrm{BBP}$ had a lower $\mathrm{pCO}_{2}$ than $\mathrm{MBP}(P=0.008)$. The concentrations of bicarbonate were lower in the BBP group compared with the MBP at $24 \mathrm{~h}$ following enrollment $(P=0.008)$. The concentration of base excess was also lower in the BBP group than $\mathrm{MBP}$ at $8 \mathrm{~h}(P=$ $0.05)$ and $24 \mathrm{~h}(P=0.01)$ following enrollment. Anion gap was found to be lower in the BBP group than the MBP group $(P=0.02)$ at $24 \mathrm{~h}$ following enrollment. No differences were found between the groups with respect to hematocrit, blood $\mathrm{pH}$, and hemoglobin. Clinical indicators of dehydration varied over the entire experiment; however, there were no differences between the groups with the exception of eye recession with calves in the 
MBP group [35\% (5/14) with no noticeable eye recession] having less severe eye recession compared with the BBP $[0 \%(0 / 16)$ with no noticeable eye recession; $P=$ $0.02]$ and HAL group $[6.7 \%(1 / 15)$ with no noticeable eye recession; $P=0.03$ at $1 \mathrm{~h}$ following enrollment. In addition, more severe eye recession was found in the HAL group at $48 \mathrm{~h}[6.7 \%(1 / 15)$ with no noticeable eye recession $P=0.04]$ following enrollment when compared with the MBP group $[21.4 \%(3 / 14)$ with no noticeable eye recession].

The results of this trial highlight that a mixed buffer solution in the treatment of calves with diarrhea led to an improved acid-base status when compared with a basic bicarbonate electrolyte powder. An important limitation to consider with this study is that the majority of calves were mildly dehydrated when treated with electrolytes. No calf mortality occurred and no calf required administration of i.v. fluids; both are indicative of minimal incidence of severe dehydration in this study. As many producers delay electrolyte therapy until dehydration is present, the use of these electrolyte products may have a different performance in a commercial setting; therefore, future studies should target calves that have more severe levels of diarrhea to identify the effect of MBP. The lack of a negative control group receiving water is another limitation to consider, as the performance of the treatment groups could not be compared with a baseline level to determine if improved control of dehydration and acidosis was achieved. A final limitation to consider is the lack of a formal sample size calculation, which may have led to an underpowered study.

Table 3. Blood gas measurements taken via i-Stat machine (Abaxis, Union City, CA) at enrollment and 1, 8 , and $24 \mathrm{~h}$ following enrollment by treatment group measured on 45 male Holstein dairy calves ${ }^{1}$

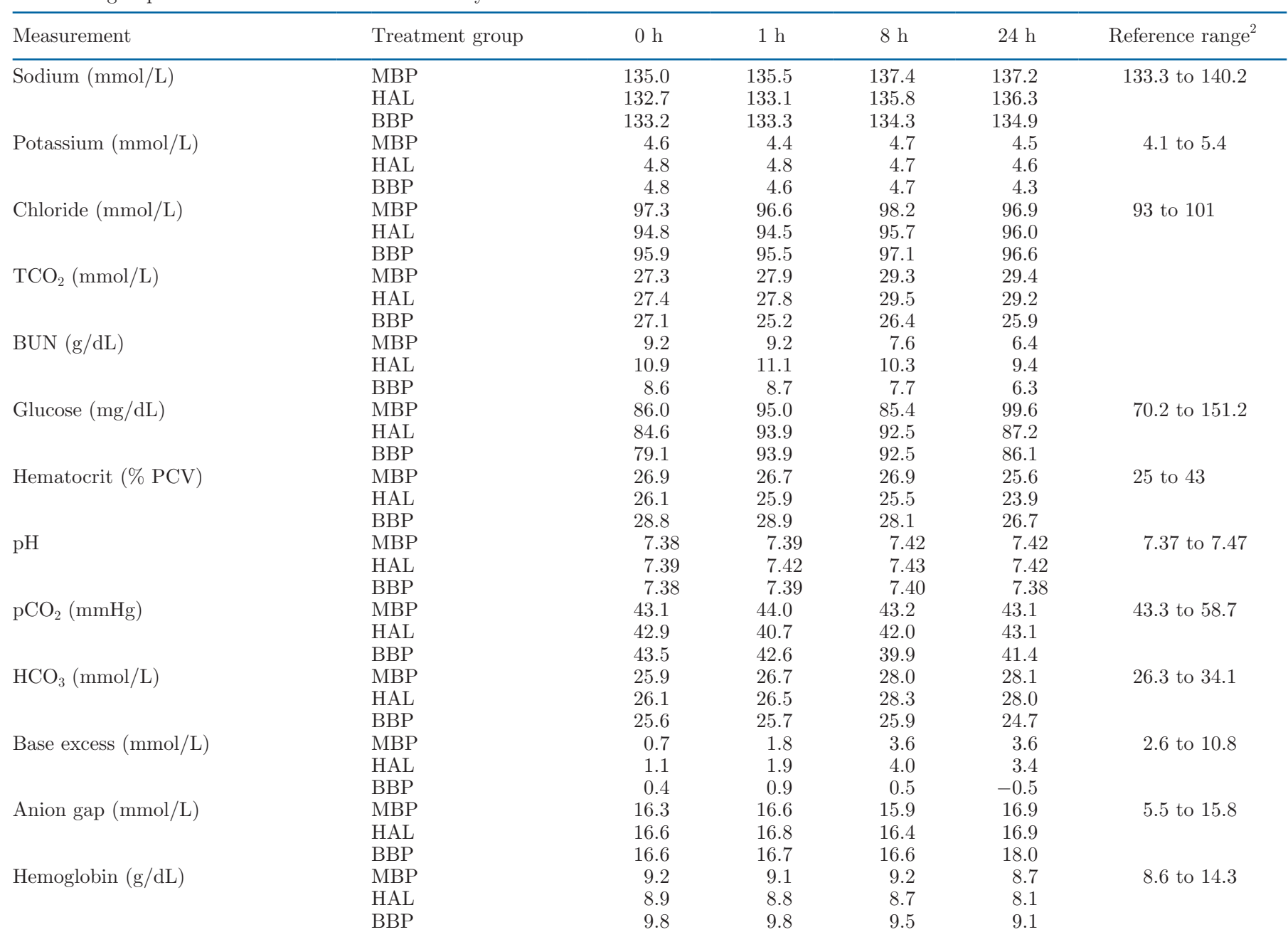

${ }^{1} \mathrm{MBP}=$ mixed buffer powder; $\mathrm{HAL}=$ high acetate liquid; $\mathrm{BBP}=$ basic bicarbonate powder; $\mathrm{TCO}_{2}=$ total carbon dioxide; $\mathrm{pCO}_{2}=$ partial pressure of carbon dioxide; PCV = packed-cell volume.

${ }^{2}$ Blood gas ranges from healthy bovine neonates (Dillane et al., 2018). 
Metabolic acidosis is characterized by a decrease in $\mathrm{pH}$ and bicarbonate concentration (Doré et al., 2019). Although blood $\mathrm{pH}$ was normal at enrollment, blood bicarbonate $\left(\mathrm{HCO}_{3}\right)$ was below reference range (Dillane et al., 2018) at $0 \mathrm{~h}$ in all 3 groups (Table 3 ), indicative that mild metabolic acidosis was present upon diagnosis of diarrhea. The MBP group improved blood bicarbonate concentrations at $24 \mathrm{~h}$ postenrollment relative to $\mathrm{BBP}$, suggestive of an improved ability to correct metabolic acidosis. This improvement is further evidenced by the improvement in base excess, total blood $\mathrm{CO}_{2}$, and anion gap at various time points by MBP when compared with BBP. There was no difference between the groups with respect to blood $\mathrm{pH}$; however, that could have been the result of the lack of a significant level of dehydration.

Daily sodium excretion in feces has been shown to increase from $3.55 \mathrm{mmol}$ in a healthy calf to $96.5 \mathrm{mmol}$ in a severe diarrheic calf, leading to a negative sodium balance (Fisher and de la Fuente, 1972). Improving sodium concentrations via OES has been shown to repair extracellular fluid volumes in diarrheic calves without predisposing the calf to hypernatremia (Michell et al., 1992). Blood sodium concentrations were maintained within normal range for healthy bovine neonates (Dillane et al., 2018) across all 3 treatment groups at 1, 8, and $24 \mathrm{~h}$ after enrollment; however, sodium concentrations were higher in MBP than in the BBP group at all 3 junctures.

Under the conditions of mild dehydration present in this study, the MBP electrolyte had similar blood buffering properties to an electrolyte composed of much higher concentrations $(7.95 \times)$ of sodium acetate (HAL), and the MBP group had mildly improved blood acid-base status as compared with BBP. Hence, an electrolyte solution composed of multiple blood buffers could be effective in correcting moderate metabolic acidosis associated with dehydration from diarrhea and is equally efficacious compared with supplementing an electrolyte with high concentrations of sodium acetate.

\section{ACKNOWLEDGMENTS}

The authors acknowledge the Mapleview Agri Ltd. (Palmerston, Canada) calf research unit staff for calf care and Rachel Genore-Roche of Acer Consulting (Guelph, Canada) for veterinary technical work. This study was funded by Animix (Juneau, WI). The authors have not stated any conflicts of interest.

\section{REFERENCES}

Bachmann, L., T. Homeier, S. Arlt, M. Brueckner, H. Rawel, C. Deiner, and H. Hartmann. 2009. Influence of different oral rehydration solutions on abomasal conditions and the acid-base status of suckling calves. J. Dairy Sci. 92:1649-1659. https://doi.org/10.3168/ jds.2008-1487.

Constable, P. D., W. Grunberg, and L. Carstensen. 2009. Comparative effects of two oral rehydration solutions on milk clotting, abomasal luminal $\mathrm{pH}$, and abomasal emptying rate in suckling calves. J. Dairy Sci. 92:296-312. https://doi.org/10.3168/jds.2008-1462.

Dillane, P., L. Krump, A. Kennedy, R. G. Sayers, and G. P. Sayers. 2018. Establishing blood gas ranges in healthy bovine neonates differentiated by age, sex, and breed type. J. Dairy Sci. 101:32053212. https://doi.org/10.3168/jds.2017-13445.

Dohoo, I., W. Martin, and H. Stryhn. 2010. Model-building strategies. Pages 365-394 in Veterinary Epidemiological Research, 2nd ed., VER Inc., Charlottetown, Prince Edward Island, Canada.

Doré, V., D. M. Foster, H. Ru, and G. W. Smith. 2019. Comparison of oral, intravenous, and subcutaneous fluid therapy for resuscitation of calves with diarrhea. J. Dairy Sci. 102:11337-11348. https://doi .org/10.3168/jds.2019-16970.

Fisher, E. W., and G. H. de la Fuente. 1972. Water and electrolyte studies in newborn calves with particular reference to the effects of diarrhea. Res. Vet. Sci. 13:315-323. https://doi.org/10.1016/S0034 $-5288(18) 34007-4$

McGuirk, S. M. 2008. Disease management of dairy calves and heifers. Vet. Clin. North Am. Food Anim. Pract. 24:139-153. https://doi .org/10.1016/j.cvfa.2007.10.003.

Medrano-Galarza, C., S. J. LeBlanc, A. Jones-Bitton, T. J. DeVries, J. Rushen, A. Marie de Passillé, M. I. Endres, and D. B. Haley. 2018. Associations between management practices and within-pen prevalence of calf diarrhea and respiratory disease on dairy farms using automated milk feeders. J. Dairy Sci. 101:2293-2308. https: //doi.org/10.3168/jds.2017-13733.

Michell, A. R., H. W. Brooks, D. G. White, and A. J. Wagstaff. 1992. The comparative effectiveness of three commercial oral solutions in correcting fluid, electrolyte and acid-base disturbances caused by calf diarrhoea. Br. Vet. J. 148:507-522. https://doi.org/10.1016/ 0007-1935(92)90006-M.

Miyazaki, T., K. Okada, T. Yamashita, and M. Miyazaki. 2019. Temporal changes of abomasal contents and volumes in calves fed milk diluted with oral rehydration salt solution. J. Vet. Med. Sci. 81:256-262. https://doi.org/10.1292/jvms.18-0466.

Naylor, J. M., L. Petrie, M. I. Rodriguez, and P. Skilnick. 1990. A comparison of three oral electrolyte solutions in the treatment of diarrheic calves. Can. Vet. J. 31:753-760.

Noël, P. G., L. Couëtil, and P. D. Constable. 2010. Effects of collecting blood into plastic heparinised Vacutainer tubes and storage conditions on blood gas analysis values in horses. Equine Vet. J. 42:91-97. https://doi.org/10.1111/j.2042-3306.2010.00248.x.

Renaud, D. L., T. F. Duffield, S. J. LeBlanc, and D. F. Kelton. 2018. Short communication: Validation of methods for practically evaluating failed passive transfer of immunity in calves arriving at a veal facility. J. Dairy Sci. 101:9516-9520. https://doi.org/10.3168/ jds.2018-14723.

Smith, G. W. 2009. Treatment of calf diarrhea: oral fluid therapy. Vet. Clin. N. Vet. Clin. North Am. Food Anim. Pract. 25:55-72. https: //doi.org/10.1016/j.cvfa.2008.10.006.

Smith, G. W., A. F. Ahmed, and P. D. Constable. 2012. Effect of orally administered electrolyte solution formulation on abomasal luminal $\mathrm{pH}$ and emptying rate in dairy calves. J. Am. Vet. Med. Assoc. 241:1075-1082. https://doi.org/10.2460/javma.241.8.1075.

Smith, G. W., and J. Berchtold. 2014. Fluid therapy in calves. Vet. Clin. North Am. Food Anim. Pract. 30:409-427. https://doi.org/ 10.1016/j.cvfa.2014.04.002.

\section{ORCIDS}

D. R. Wood ๑ https://orcid.org/0000-0003-0799-0361

G. W. Smith @ https://orcid.org/0000-0001-5700-8810

D. L. Renaud () https://orcid.org/0000-0002-3439-3987 